\author{
A.A. Shavrov (Junior), A.E. Alexandrov, I.V. Kirgizov, A.A. Shavrov, A.Y. Kharitonova, \\ G.V. Volynets, A.G. Talalaev \\ Scientific Center of Children’s Health, Moscow, Russian Federation
}

\title{
Intravenous fluorescein administration safety at confocal laser endomicroscopy of digestive tract in children
}

\section{Author affiliation:}

Anton Andreevich Shavrov, endoscopist of the endoscopy department at the research institute of pediatrics of the FSBI "SCCCH"

Address: Moscow, Lomonosovskiy Av. 2/1, 119991, tel.: (499) 134-04-12, e-mail: shavrovnczd@yandex.ru

Article received: 17.02.2013. Accepted for publication:

Confocal laser endomicroscopy (CLE) is a method of optic biopsy, which allows obtaining histologic presentation of mucous tunic in real time and quickly differentiating between unaltered and inflamed, metaplastic and dysplastic tissue structures, thus developing a new and safe method of early diagnostics of diseases. Intravenous administration of $10 \%$ sodium fluorescein is necessary in order to obtain high-contrast images of the structure of digestive tract's mucous tunic, although there have been no direct indications to that in the world pediatric practice.

The authors examined 124 children of 3-18 years of age who received intravenous fluorescein of $5 \mathrm{mg} / \mathrm{kg}$. No severe side effects were revealed at intravenous administration. The authors revealed temporary yellowing of skin and bright coloration of urine. In terms of mild side effects, the authors observed insignificant nausea and emesis in 2 children (1.6\%). Strict observation of the selection protocol allows not only avoiding severe complications caused by administration of the drug, but also recommending its use at CLE of digestive tract's mucous tunic in children.

Keywords: confocal laser endomicroscopy, pediatrics, fluorescein, CLE.

\section{Introduction}

Detailed visualization of the digestive tract's mucous tunic is necessary for early diagnostics of a disease. Probe-based confocal laser endomicroscopy (pCLE) is a new method of gastrointestinal endoscopy, which yields images of mucous tunic of ca. 1 micron resolution and visualizes cellular and subcellular structures, capillaries and singular blood cells. The available published data indicate high interconnection of confocal endomicroscopy intravital images with the traditional histology of the unaltered digestive tract's mucous tunic [1, 2]. The technique allows quickly differentiating between an inflamed, metaplastic or dysplastic mucous tunic; without any doubt, this increases interest in the use of this technique in clinical practice for diagnosis of such diseases as Barrett esophagus, gastric metaplasia of esophagus, neoplasms, inflammatory diseases of large and small intestines, Helicobacter pylori, celiac disease etc.

It should be noted, that it is necessary to administer a diagnostic agent intravenously in order to obtain high-contrast images at pCLE of digestive tract. 10\% sodium fluorescein solution may be used; it is widely applied in diagnostic practice in adult patients [2, 3]. In 2008, the Ministry of Health of the RF approved intravenous fluorescein administration for evaluation of eye fundus condition using a confocal laser ophthalmoscope (Registration number LRS-000829/08 of 18.02.08); the procedure itself appeared to be of excellent safety level in pediatric ophthalmology [3]. This study is relevant due to the lack of direct indication in the world pediatric practice to intravenous administration of this PCLE-required diagnostic agent.

\section{Patients and methods}

We examined 124 children (73 boys, 51 girls) of 3-18 years of age treated at the clinic of the FSBU “SCCH” Speranskiy research institute of pediatrics from October 2010 to January 2013. 
All children underwent endoscopic examination of digestive tract supplemented with pCLE with intravenous administration of $5 \mathrm{ml}$ per $1 \mathrm{~kg}$ of body weight of the $10 \%$ sodium fluorescein solution (Novartis, Hiningue, France). Observational trials were approved by the local independent Ethical committee of the Center and Scientific Council of the FSBU "SCCH". Repeated intravenous administration of the diagnostic agent to older children was permitted in case of none or only minimal side effects. We also received informed consent to the procedure of all patients or their parents. Renal and hepatic failure and allergic reactions to drugs stated in the anamnesis were the main contraindications to the agent's administration. It is common knowledge that, upon entrance to blood channels, $80 \%$ of diagnostic agent fluorescein is bonded by blood serum albumins; it spreads via capillaries, penetrates tissues and colors enterocytes, superficial epithelium's cytoplasm, membrane, crypts, infiltrating cells and blood vessels for a period of 30 minutes; it does not color cell nucleus and mucin, so they appear dark. Liver quickly metabolizes the agent into fluorescein monoglucuronide, which is later discharged by kidneys [5].

We observed whether severe or insignificant side effects would appear in patients after intravenous administration of the diagnostic agent. We considered a complication severe, if it was life-threatening. All other complications were considered insignificant. During the procedure (once in 5 minutes during the trial and once in 15 minutes within 2 hours after the manipulation), we supervised vital functions using a Datex Ohmeda Cardiocap 5 monitor (Denmark): pulse oximetry, arterial pressure measurement, electrocardiography, thermometry. All trials were conducted under general sevoflurane anesthesia, standard technique. We monitored the patient's condition within 2 hours after the agent's administration at the endoscopic department's post-anesthesia care unit according to the aforementioned scheme.

Before transfer of patients, their attending doctors had to indicate presence or absence of side effects. Parents were advised to record side effects themselves within 1 month after the procedure after discharge of their children. Criteria of side effects included systemic allergic reactions of any type, rash, pruritus, labored breathing, crepitation and any other symptoms, of which patients or parents complained.

\section{Results and discussion}

We did not reveal any severe side effects in our series of observations of 124 children. According to the available published literature, anaphylactic shock in response to intravenous fluorescein administration during eye fundus angiography is an extremely rare complication (1 out of 22,000 patients). Allergy established in the anamnesis of several patients and need in a diagnostic trial implied prescription of desensitizing therapy [6, 7]. According to other data, allergic reactions resulting in cardiopulmonary failure have never been registered at confocal laser endomicroscopy.

The most frequent side effects in our series of observations were short-term yellowing of skin and visible mucous tunics and bright yellow urine coloring, which disappeared within 2-3 hours. Insignificant nausea and retching were observed in 2 children (1.6\%). However, we were unable to link this side effect to intravenous administration of the agent, as it might have served as a manifestation of general anesthesia. Mild nausea and emesis were observed in 2-10\% of ophthalmologic practice cases in adults [8,9]; they appeared immediately after the agent's administration $[6,10]$.

Other authors mentioned short-term hypotension, injection site inflammation, self-limited rash and insignificant epigastric pains in adult patients at pCLE [3]. We did not observe this type of side effects at pCLE in our series of observations (tb.). Overall, minimal side effects in response to intravenous fluorescein administration (dosage $-5 \mathrm{mg} / \mathrm{kg}$ ) under general anesthesia may be linked to correct selection of patients for probe-based confocal laser endomicroscopy. 
Table. Side effects at intravenous fluorescein administration

\begin{tabular}{|l|l|}
\hline Insignificant side effects & Amount \\
\hline Skin rash & 0 \\
\hline Nausea, emesis & $2(1.6 \%)$ \\
\hline Abdominal pain & 0 \\
\hline Hemodynamic disorders & 0 \\
\hline Severe side effects & 0 \\
\hline
\end{tabular}

\section{Conclusion}

Intravenous administration of 10\% sodium fluorescein solution (5 mg per $1 \mathrm{~kg}$ of body weight) at pCLE of digestive tract in children of 3-18 years of age may be considered safe. Use of this diagnostic agent is necessary to obtain high-contrast pCLE images; in its turn, pCLE increases precision of early diagnostics of diseases by differentiating mucous tunic's structure. Our trial showed that side effects at intravenous fluorescein administration are extremely rare and mostly insignificant.

Strict observation of the trial selection protocol allows preventing severe complications in response to the agent's administration and recommending it for use at pCLE in pediatric practice.

\section{REFERENCES}

1. Venediktova M. M., Shavrov A. A. Voprosy diagnostiki v pediatrii-diagnostics in pediatrics. 2012; 4: 17-21.

2. Wallace M. B., Fockens P. Probe-based confocal laser endomicriscopy. Gastroenterology. 2009; 136: 1509-13.

3. Becker V., von Delius S., Bajbouj M., Karagianni A., Schmid R. M., Meining A. Intravenous application of fluorescein for confocal laser scanning microscopy: evaluation of contrast dynamics and image quality with increasing injection-to-imaging time. Gastrointest Endosc. 2008; 68: 319-23.

4. Markova E. Yu. Differentsial'naya diagnostika i lechenie zabolevanii glaznogo dna u detei. Avtoref. dis. ... dokt. med. nauk [Differential Diagnosis and Treatment of Diseases of the Fundus in Children. Author's abstract]. Moscow, 2008. P. 55.

5. Mandava N., Reichel E., Guyer D. Fluorescein and ICG Angiography, 2nd edn. St Louis: Mosby. 2004; 106: 800-808.

6. Yannuzzi L. A., Rohrer K. T., Tindel L. J. et al. Fluorescein angiography complicationsurvey. Ophthalmology. 1986; 93: 611-7.

7. Kwan A. S., Barry C., McAllister I. L., Constable I. Fluorescein angiography and adverse drug reactions revisited: the Lions Eye experience. Clin experiment ophthalmol. 2006; 34: 33-8.

8. Lopez-Saez M. P., Ordoqui E., Tornero P. et al. Fluoresceininduced allergic reaction. Ann Allergy Asthma Immunol. 1998; 81: 428-30.

9. Jennings B. J., Mathews D. E. Adverse reactions during retinal fluorescein angiography. $J$ Am Optom Assoc. 1994; 65: 465-71.

10. Moosbrugger K. A., Sheidow T. G. Evaluation of the side effects and image quality during fluorescein angiography comparing $2 \mathrm{~mL}$ and $5 \mathrm{~mL}$ sodium fluorescein. Can J Ophthalmol. 2008; 43: 571-5. 